Omar H. Alluazy

BDs, MSc (Lec.)

Hakam H. Sabah

BDs, MSc (Lec.)

Saba H. Al-Zubaidi

BDs, MSc (Lec.)

Mustafa M. Al-Sultan BDs, MSc (Asst. Prof)

\section{A Comparative Assessment of Space Closure Utilizing by Lingual and Con- ventional Brackets}

Depart. of Pedod, Orthod and Preve.Dentistry

College of Dentistry, University of Mosul

Depart. of Pedod, Orthod and Preve.Dentistry

College of Dentistry, University of Mosul

Depart. of Pedod, Orthod and Preve.Dentistry

College of Dentistry, University of Mosul

Depart. of Pedod, Orthod and Preve.Dentistry

College of Dentistry, University of Mosul

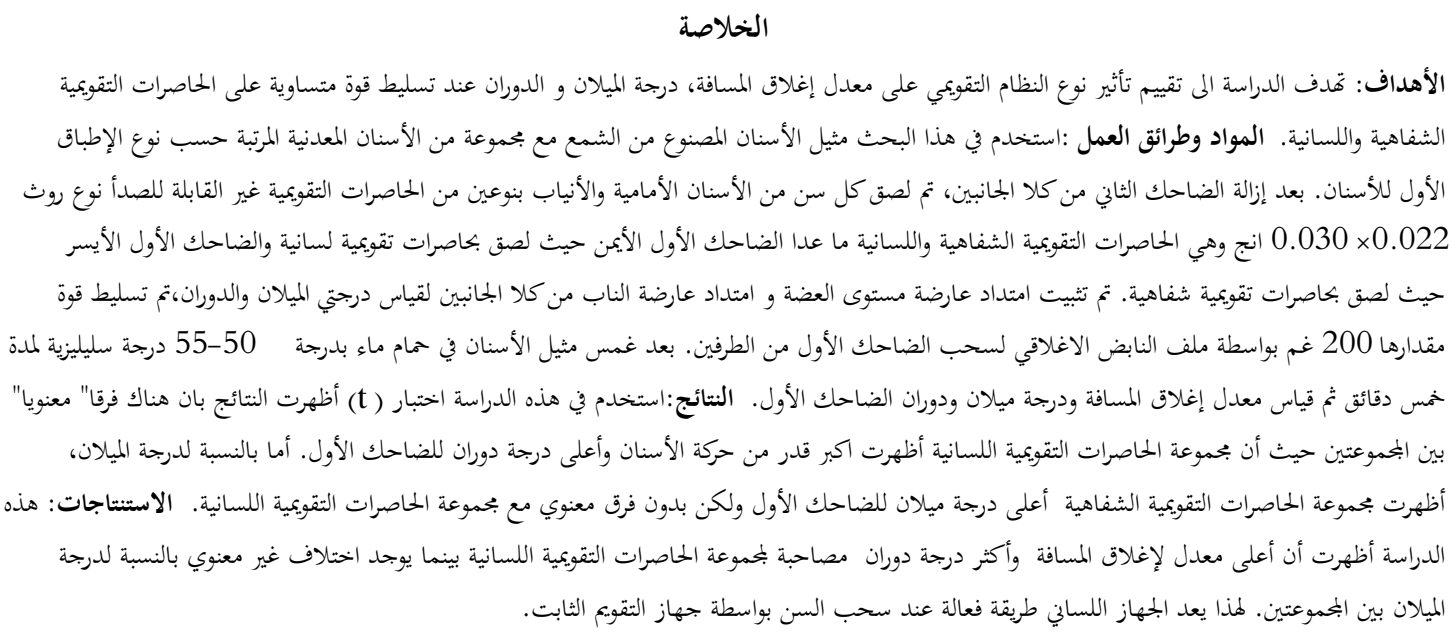

ABSTRACT

AIMS:To assess the effect of system type on the amount of space closure when equal force use on each system and to compare the amount of rotation and tipping generated by lingual and conventional brackets. Materials and Methods: A mandibular typodont system with Class I wax form and set of metal teeth were used in this study. After removing the second premolar bilaterally, each tooth of the anterior teeth and canines were bonded with two bracket systems, preadjusted Roth stainless steel 0.022x0.030 inch conventional (labial) brackets (CoBS group) and lingual brackets (LiBS group) except right first premolar were bonded with lingual bracket (LIBS group) and left first premolar were bonded with labial bracket (CoBS group). Bite plane extension bar (BPB) and canine extension bar (CB) were constructed bilaterally for the measurement of first premolars tipping and rotation. A $200 \mathrm{gm}$. of force applied by close coil spring to retract the first premolars bilaterally . After immersion of the typodont in water bath with 50-550 C for 5 minutes, the rate of space closure, tipping and rotation of first premolars were measured. Results: A statistical analysis (independent-samples t-test) used in this study, there was a significant difference between the two system groups (CoBS group and LiBS group). The LiBS group had a significant higher amount of tooth movement, and higher degree of rotation. For the tipping, the CoBS group had the higher level of tipping with a non-significant difference with LiBS group. Conclusions: The outcomes of this study showed that the higher rate of space closure and rotation were associated with LiBS group, while there is a nonsignificant difference in the amount of tipping between the two groups. So, Lingual appliance was appeared to be very effective method for tooth sliding during fixed orthodontic treatment.

Keywords: Lingual bracket, conventional bracket, sliding and typodont.

Alluazy OH, Sabah HH, Al-Zubaidi SH, Al-Sultan MM,A .Comparative Assessment of Space Closure Utilizing by Lingual and Con-ventional Brackets. Al-Rafidain Dent J. 2014; 14(2):220-227.

Received: 18/11/2013 Sent to Referees: 26/11/2013 Accepted for Publication: 23/2/2014 


\section{INTRODUCTION}

Recent years have seen a marked increase in number of adult patients desiring orthodontic treatment, it is well known that the adult have negative reaction toward the esthetic of conventional fixed orthodontic appliance. Even through brackets made of plastic and porcelain and coated arch wire. ${ }^{(1)}$ So, the only solution that provides ultimate method of esthetics during treatment is attach the appliance to the lingual surface of teeth. ${ }^{(1)}$ However, the "lingual" appliance is superior to traditional "labial" appliance and lingual appliance is covering the same range of treatment indication as do labial devices but the patient that is reported greater speech disturbance, pain, cost and irritation of tongue. ${ }^{(2-4)}$ Typodont can be used in orthodontic practice to show possible effect of using variable factors of canine position and rate of movement during sliding mechanics. ${ }^{(5)}$

The orthodontic diagnosis and treatment plan often required retraction of canines, where the retraction of canine consider the basic technique in orthodontic treatment. ${ }^{(6,7)}$ The common method of translating tooth orthodontically was done by the use of sliding mechanics and mesiodistal tooth movement is accomplished by guiding the tooth along continuous arch wire with help of an orthodontic bracket in sliding mechanics ${ }^{(8-10)}$, position of bracket give control tooth movement. ${ }^{(11)}$ Pure bodily distal movement of canine is difficult to achieve with so-called sliding

mechanics. ${ }^{(12)}$ Any force to achieve desired tooth movement must exceed the friction force inherent the appliance. ${ }^{(13)}$ Frictional forces act in opposite direction to the desired tooth movement and generated when a force applied to bodies in contact.Canine tipping during initial unsteady stat then moved bodily during steady stat and tipping of canine decreased when wire sized increased or when force applied decreased. ${ }^{(7,8)}$ Clearance between the arch wire and bracket slots there will be considerably more tipping also increase friction resistance as binding occur between bracket slot and arch wire and degree of tipping with sliding mechanics would probably smaller if rectangular arch wire had used and tipping tooth movement is smaller if the surface force applied close to the neck of tooth or more gingivally. ${ }^{(14)}$ Canine will rotate if the force applied not through the center of resistance of tooth labiolingually direction. ${ }^{(9)}$

The aims of this study compare the amount of rotation, tipping and space closure between the lingual and conventional brackets.

\section{MATERIALS AND METHODS}

The study was conducted using typodont model, with wax form mandibular class I, a set of mandibular metal teeth (all teeth except third molars), the typodont was prepared according to manufacturer's instructions (Ormco, Japan), the preadjusted Roth stainless steel conventional (labial) brackets 0.022x0.030 inch (Denturum-Germany) and lingual brackets $0.022 \times 0.030$ inch (SMART-china) were fixed on the labial and lingual surfaces of metallic typodont teeth (Figure 1) by using epoxy steel adhesive (USA). ${ }^{(15)}$

The lingual bracket fixed on the lingual surface of all metal teeth except the left first premolar, while the conventional brackets fixed on the labial and buccal surface of all metal teeth except the right first premolar, the preadjusted stainless steel buccal bondable tube (hallimexgermany) were fixed to the buccal surface of molars teeth bilaterally, also preadjusted stainless steel lingual bondable tube (SMART-china) were fixed to the lingualsurface of molars teeth bilaterally.

Alignment of the teeth was done by using arch wires started from 0.014 inch round NiTi (Denturum, Germany) and gradually upgraded till reached $0.016 \times 0.022$. Rectangular stainless steel conventional and lingual arch wires ${ }^{(16,17)}$, arch wires were ligated to the brackets by ligature wire 0.010 inch (ISO-USA) in both lingual and conventional brackets . The second right and left premolars where removed from typodont to allow a space for first premolar sliding.

After that, a well aligned L shape round wire 0.1 inch adapted to the occlusal surfaces of first and second molars and extended to the distal surface of second molar on both side of lower arch, then fixed in position by using epoxy steel 
adhesive. This L shape wire hold the two molars firmly together and to the metal base of the typodont to make them immobile (Figure 1).

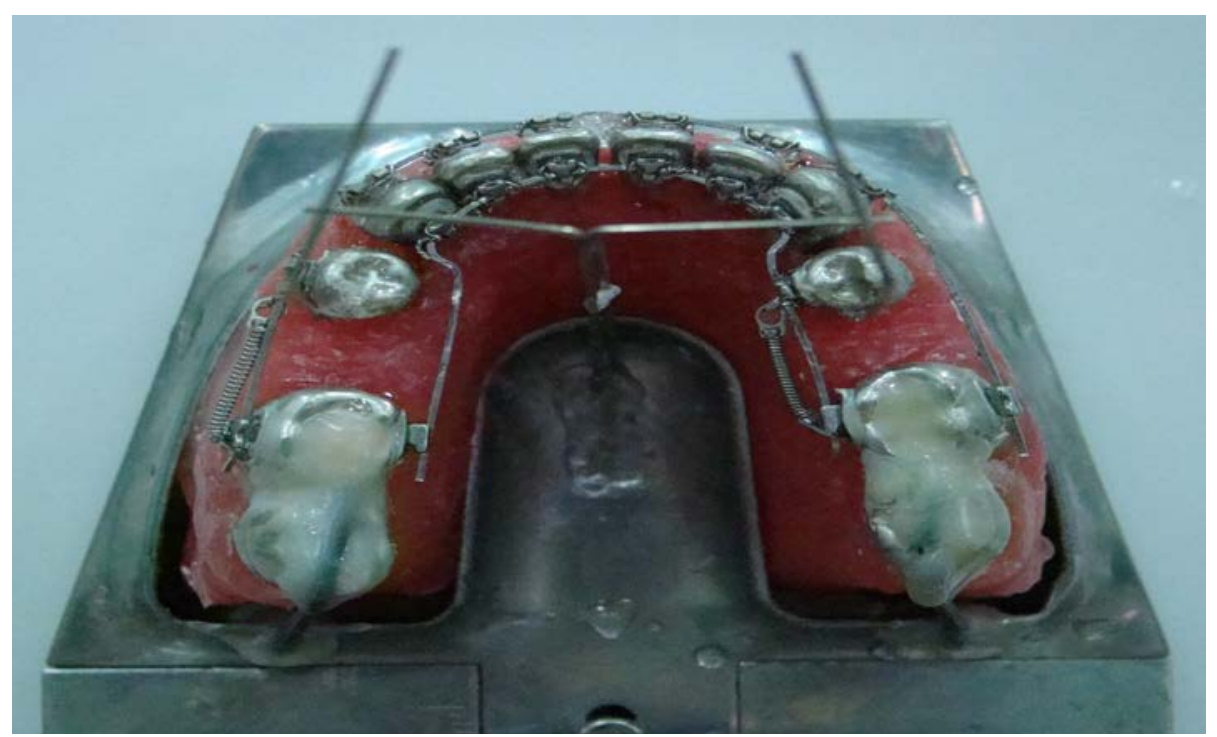

Figure (1): Occlussal view shows Closed coil spring between the lower first premolars (CoBS left side and LiBS right side) and the hook at the first molars band after immersing in water bath

A special four bars were constructed, 2 bilateral bite plane extension bars (BPB) and 2 bilateral first premolars extension bars (CB) (Figure 2), the 2 BPB were L-shape bars made from $0.016 \times 0.016$ inch stainless steel rectangular wire, the long arms were inserted and fixed to the typodont by using epoxy steel adhesive in the simulated tongue position area in the midline and in the same level of the premolars, those bars emerges upward for $(50 \mathrm{~mm})$ then it bends and extended facially $(30 \mathrm{~mm})$ to make right angle with $\mathrm{CB}(9)$, the $2 \mathrm{CB}$ were also constructed from $0.016 \times 0.016$ inch stainless steel rectangular wire in a form of L-shape, the long arms were fixed to the distal aspects of the first premolars ( the teeth to be slides) by using epoxy steel adhesive, those arms extended upward incisally $60 \mathrm{~mm}$ then they bend at right angle and extended anteriorly $40 \mathrm{~mm}$ and above the BPB by about $20 \mathrm{~mm}$. Such two bars in each side are used as a guide to determine the position of first premolar after sliding regarding the degree of tipping and rotation, this method is a modification of Huffman and Way procedure. ${ }^{(10)}$ A special wooden table was constructed with two metallic bases, one to fix the digit camera (vertically for rotation measurement and horizontally for tipping measurement), and the other to fix the typodont in a way to allow a standard method for taking a photograph before and after each sliding in both right side sliding (lingual bracket system ) and left side sliding (conventional bracket system). 


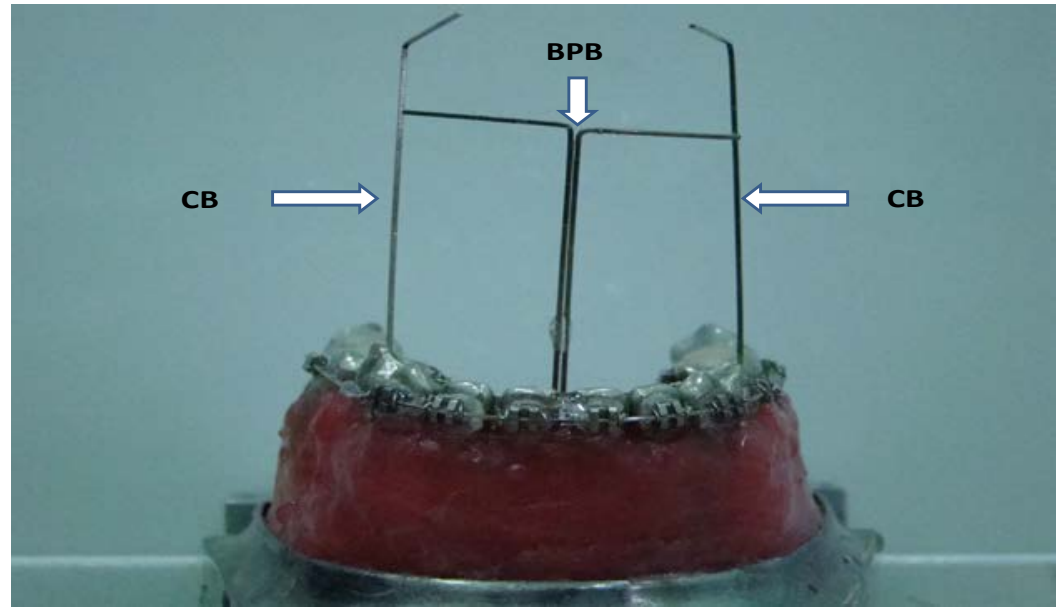

Figure(2): Frontal view shows the CB and BPB bars after immersing in water bath.

In this study, two systems were used for sliding the first premolar distally, the first system is the Conventional Bracket system (CoBS) group in the left side and the second system is the lingual bracket system (LiBS) group in the right side, the retraction of first premolars in the two side were done by using close coil spring (Denturum-Germany), with force $200 \mathrm{gm}$ using tension gauge (France) to measure the delivered force in both side (CoBS group and LiBS group).(18) The typodont was immersed in a water bath (HAAKE-England) with 50-55 0C for 5 minutes then removed and immersed directly in a tap water (5 -10 C0 )(19), this procedure repeated with each measurement .

\section{Before sliding:}

In this study, the first premolar slide bilaterally 10 times by immersing the typodont in the water bath, before each method of sliding, the distance between the distal wing of left first premolar's bracket(CoBS) and the mesial end of the first molar's tube was measured, also the distance between the distal surface of right first premolars bracket (LiBS) and the mesial end of the first molar's lingual tube was measured. This distance is considered as the available space, This distance was measured in millimeter by digital vernia (Sony-china), also the angle between BPB bars and CB bars should be 90 from both horizontal and vertical direction in both left and right sides; these angle is considered as CB original angle.
After sliding:

After each method of first premolar sliding bilaterally by immersing the typodont in the water bath, the distance between the distal surfaces of first premolars brackets (CoBS and LiBS) and the mesial end of the first molar's tube were again measured(20) by digital vernia, this distance is considered as the remaining space, therefore:

Rate of space closure=Available spaceRemaining space

The first premolars tipping degree in the two groups after sliding were measured by taking a photograph to the typodont using digital camera(canon , Japan), with horizontal project from right and left side (directed towered first premolar) where the angle between $\mathrm{BPB}$ and $\mathrm{CB}$ are exposed and then can be measured directly on the photograph using protractor, this angle is considered as $\mathrm{CB}$ inclination angle from the $\mathrm{CB}$ original angle, then the first premolar's tipping were measured by subtraction of the $\mathrm{CB}$ tipping angle from the $\mathrm{CB}$ original angle. The first premolars rotation degree in the two groups after sliding were measured by taking a photograph to the typodont using digital camera, with vertical project from occlusal side (directed towered the center midline of the typodont) where the angle between BPB and CB are exposed and then can be measured directly on the photograph using protractor, then the first premolar's rotation were measured by subtraction of the CB rotational angle 
from the CB original angle.

\section{RESULTS}

The descriptive statistics analysis, (minimum, maximum, mean value ,standard error and standard deviations) for the rate of space closure, tipping and rotation for both conventional bracket system (CoBS) group and lingual bracket system(LiBS) group, are given in (Table 1). The independent-samples t-test (Table 2) was carried out for rate of space closure, rotation and tipping to compare between (CoBS) group and (LiBS) group.

Table (1): Descriptive Statistics Demonstrating the Effect of Groups on Shear Bond Strength of Brackets.

\begin{tabular}{cccccccc}
\hline & Groups $^{*}$ & No. & Minimum & Maximum & mean & SD & Std. Error \\
\hline $\begin{array}{c}\text { Rate of } \\
\text { space }\end{array}$ & CoBS & 10 & 1.00 & 3.00 & 1.915 & .6774 & .2142 \\
closure & LiBS & 10 & 3.20 & 6.20 & 4.380 & .8390 & .2653 \\
Rotation & CoBS & 10 & 1.00 & 3 & 2.240 & .6653 & .2104 \\
& LiBS & 10 & 2.00 & 6.50 & 4.390 & 1.571 & .4969 \\
Tipping & CoBS & 10 & .50 & 1.70 & 1.000 & .4320 & .1366 \\
& LiBS & 10 & .50 & 1.80 & .9900 & .4863 & .1538 \\
\hline
\end{tabular}

* COB: conventional bracket system, LiB: lingual bracket system.

Table (2): Comparison between conventional bracket system group and lingual bracket system group by using t-test for Rate of Space Closure, Rotation and Tipping.

\begin{tabular}{|c|c|c|c|c|c|c|}
\hline & Groups & Mean & \pm SD & t - Value & Df & p-Value \\
\hline Rate of space & CoBS & 1.915 & .6774 & & & \\
\hline closure & LiBS & 4.380 & .8390 & -7.228 & 18 & $.000^{*}$ \\
\hline Rotation & $\begin{array}{l}\text { CoBS } \\
\text { LiBS }\end{array}$ & $\begin{array}{l}2.240 \\
4.390\end{array}$ & $\begin{array}{l}.6653 \\
1.571\end{array}$ & -3.984 & 18 & $.001 *$ \\
\hline Tipping & $\begin{array}{l}\text { CoBS } \\
\text { LiBS }\end{array}$ & $\begin{array}{l}1.000 \\
.9900\end{array}$ & $\begin{array}{l}.4320 \\
.4863\end{array}$ & 0.49 & 18 & 962 \\
\hline
\end{tabular}

* Significant differences existed at $\mathrm{p} \leq 0.05$

Rate of space closure

The t-test for the rate of space closure showed that there was a significant difference between CoBS group and LiBS group at $\mathrm{p} \leq 0.05$ as shown in (Table 2). LiBS group was higher mean value of 4.380 if compared to CoBS group which was 1.9150 .

\section{Rotation}

After subtraction of the $\mathrm{CB}$ rotational angle from the CB original angle, in CoBS group the degree of angle was positive while in the LiBS group was negative. Because in the CoBS group first premolar was rotated mesiolingually or Distolabially, while in the LiBS group first premolar rotated mesiolabially or distolingually.

The t-test for the rotation showed that there was a significant difference between CoBS group and LiBS group at $\mathrm{p} \leq$ 0.05 as shown in (Table 2). LiBS group was higher mean value of 4.390 if com- pared to CoBS group which was 2.240 Tipping

The t-test for the tipping showed that there was no- significant difference between CoBS group and LiBS group at $\mathrm{p} \leq 0.05$ as shown in (Table 2). CoBS group was higher mean value of 1.000 if compared to LiBS group which was .9900

\section{DISSCUSION}

The mechanical properties of the bracket-wire-interface influences the efficiency of any fixed appliance, especially the lingual appliance. Up now few studies have looked into mechanical properties of the bracket-wire interface. ${ }^{(21)}$ In this study, a comparative assessment of space closure, amount of rotation and tipping generated by lingual and conventional brackets.

According to the result of Goren etal. ${ }^{(22)}$ In both systems, during retraction 
of the first premolar, the direction of the force applied passes relatively far from the centre of resistance, and therefore, moment is created. The moment tends to move the crown in the force direction and the apex in the opposite direction. In this sense, there is no difference between CoBS and LiBS in regard to the tipping of the tooth. This result come in agreement with our result .Since tipping will occur until contact is established between the diagonal corners of the bracket slot and arch wire, so that the low mean value of the tipping result between the CoBS and LiBS may be due to the used of the large arch wire $(0.016 \times 0.022 "$ stainless steel) and the non significant tipping that result between the CoBS and LiBS may be due to the same arch wire $0.016 \times 0.022 "$ stainless steel used in this study.

The sagittal force also creates a moment in the bucco-lingual direction, which tends to rotate the tooth in a disto-lingual direction when CoBS is used, and meso- buccal rotation of the tooth when LiBS is used. which comes in agreement with the result obtained by Goren etal ${ }^{(22)}$ as they stated that, in buccal orthodontics, the vector of force passes buccal to the centre of resistance and in lingual orthodontics, it passes lingual to it. therefore, the direction of these rotations are opposite.

The wider the mesio-distal width of the CoBS result in good control of the arch in the bracket slot, this explain the significant less rotation that occure in CoBS when compare with LiBS. This result come in agreement with other studies. ${ }^{(23,24)}$ Therefore, Fuck etal (25) recommended the use of double over tie in lingual techniques to better control of the arch wire within the narrow bracket's slot .

When the brackets are placed on the lingual surface instead of the labial surface, the inter-bracket distance is decrease lingually, and this makes the wire stiffer in lingual orthodontic than in labial orthodontic. ${ }^{(26)}$ Stiffer wires reduce the binding and thus reduce the resistance to friction. ${ }^{(11,27)}$ Also, the smaller mesio-distal width of the LiBS in comparison with the CoBS reduce the contact area with the arch wire, and subsequently reduce the friction. This explains why in our study the rate of space closure was significantly greater in LiBS than in CoBS. This result comes in agreement with other studies. ${ }^{(22,25)}$

Friction is created between the arch wire and the bracket for two main reasons, ${ }^{(28)}$ the first is the possible pull of the ligature, the second is more complicated to understand but inevitable. It is induced by the task of the bracket to prevent undesirable tipping and rotation or depending on the task-the intentional application of a derotation moment.

\section{CONCLUSIONS}

Lingual orthodontic appliances represent anssz excellent alternative to labial appliances for patients. The mechano therapy involved is altered in some respects.

In our study, LiBS group showed significantly more rotation and greater amount of space closure during retraction of the first premolar when compare with COBS, so this study recommended the use of lingual system when bodily movement of tooth with less force and less amount of anchorage lost are wanted.

\section{REFERENCES}

1. Cem C, Yildis O . Patient discomfort: A comparison between lingual and labial fixed appliance. Angle Orthod. 2005;75(1):86-91.

2. Lars-M F, Dirk W, Oicter D. Comparison of initial orthodontic force systems produced by a new lingual bracket system and straight-wire appliance. $J$ OrofacialOthop. 2005; 66(5):363-376.

3. Burak A, Cem C, Yildis O. Comparison of bond strength of lingual brackets under different composite base forming method. Hellenic Orthod Review. 2005; 8(2):81-91.

4. Yildis O, Tugce YA, Bulent A. Comparative in vitro study of frictional resistance between lingual brackets and stainless steel arch wires. Eur. $J$ Orthod. 2012;34(1):119-125.

5. Loftus BP, Artun J, Nicholls JI, Alonzo TA, Stoner JA. Evaluation of friction during sliding tooth movement in various bracket arch wire combination. Am J Orthod Dentofacial 
Orthop.1999; 116(3): 363-542.

6. Miri S, Rena Ck, InbalI,Laura C, Boaz T,Eytan B, Stella C. Adult patient's adjustability to orthodontic appliance. Part 1 A comparsion between labial,lingual and invisalign . Eur J Orthod. 2012;34(6):724-30.

7. Mauricio M, Eduardo S D, Luciane M de Menezes, Andre W, Susiane A. Maxillary canine retraction with self- ligating and conventional brackets. Angle Orthod. 2011; 81(2): 292-297.

8. Syedaltaf K, Vadivd K, Prithviraj J. The comparison of friction resistance in titanium resistance in titanium, self-ligating stainless steel bracket using stainless and TMA arch wire. $J$ pharm Bioallied Sci. 2012;(4) suppl 2: 203-211.

9. Choy K, Pae E, Kim K, Park Y, Burstone C. Control space closure with statically determinate retraction system. Angle Orthod. 2002; 72(3): 191-198.

10.Sueri MY, Turk T. Effectiveness of laceback ligatures on the maxillary canine retraction. Angle Orthod. 2006;76(6): 1010-1014.

11.Huttman DJ, Way DC. A clinical evaluation of tooth movement along arch wire of two different sizes. Am J Orthod Dentofcial Orthop 1983; 6:453459.

12.Ziegler P, Ingervall B. A clinical study of maxillary canine retraction with a retraction spring and with sliding mechanics. Am J Orthod. Dento facial Orthop.1989; 95(2): 99-106.

13.Hain M, Ohopatkar A, Roch P. The effect of ligation method on friction in sliding mechanics. Am $J$ Orthod Dentofacial Orthop, 2005; 123(4) : 416-422.

14.Senka M , Mladen S, Petra R . Finite element method analysis of tooth movement induced by orthodontic forces. CollAntropol. 2003; (27) suppl 2: 17-21.

15. Mulaughlin RP , Bannett JC. Bracket placement with the preadjusted appliance. J Clin. Orthod. 1995; 29(5):302-311.

16.Al-Shahery WG. Upper canine retraction by sliding technique using stainless steel and nickel titanium coil springs (A comparative study in vitro ) : MSc. Thesis, collage of dentistry, university of Mosul. 2010;64.

17.Eliades T, Boourauel C . Intraoral aging of orthodontics material:the picture we miss and its clinical relevance. Am J OrthodDentofacialOrthop. 2005;127(4):403-412.

18.Quinn RS, Yoshikawa Dk . A reassessment of force magnitude in orthodontics. Am J Orthod .1985; 88(3):252-60.

19.Lima V, Coimbra M , Derech C. Frictional forces in stainless steel and plastic brackets using four types of wire ligation. Dent Press J. Orthod. 2010; 15(2):82-86.

20.Sonis AL. Comparison of nickel-titanium coil springs vs. elastics in canine retraction. $J$ ClinOrthod. 1994; 28(5):145-148.

21.Silvia G, Rafi R , Pablo E. Adult and lingual orthodontics. Paper was presented at the 2nd international conference of the JLOA in Tokyo. 2007; 5(1).

22.Goren S, Zoizner R, Geron S, Romano. Lingual orthodontics (LO) versus buccal orthodontics (BO). Biomechanical and clinical aspects. $J$ LlingualOrthod .2012; 3(1):1-7.

23.Demicheri R: Rotated teeth-effective treatment utilizing the lingual technique. Ortho tribune. The Wolds orthodontic newspaper. US edition. 2009.

24.Park J, Lee $Y$, Lim B, Kim C . Frictional forces between lingual brackets and arch wires measured by a friction tester. Angle Orthod. 2004; 74(6):816-824.

25.Fuck L, Wiechmann D, Drescher D. Comparison of the initial orthodontic force systems produced by a new lingual bracket system and a straightwire appliance. $J$ Orofacial Orthop. 2005; 66(5):363-76.

26. Moran Kl. Relative wire stiffness due to lingual versus labial inter bracket distance. Am J Orthod Dento facial Orthop. 1987;92(1):24-32. 
27.Dresher D, Bouraull C, Schumacher H . Frictional forces between bracket and arch wire. Am J Orthod Dentofacial. Orthop. 1989; 96(5):397-404.
28.Segner D, Ibe D. Properties of super-elastic wires and their relevance to orthodontic treatment. Eur $J$ Orthod. 1995; 17(5):395-402. 